\title{
The First Report of Mycobacterium celatum Isolation from Domestic Pig (Sus scrofa domestica) and Roe Deer (Capreolus capreolus) and an Overview of Human Infections in Slovenia
}

\author{
Mateja Pate, ${ }^{1}$ Manca Žolnir-Dovč, ${ }^{2}$ Darja Kušar, ${ }^{1}$ Brane Krt, ${ }^{1}$ Silvio Špičić, ${ }^{3}$ \\ Željko Cvetnić, ${ }^{3}$ and Matjaž Ocepek ${ }^{1}$ \\ ${ }^{1}$ Veterinary Faculty, University of Ljubljana, Gerbičeva 60, 1115 Ljubljana, Slovenia \\ ${ }^{2}$ National Reference Laboratory for Mycobacteria, University Clinic for Respiratory and Allergic Diseases Golnik, Golnik 36, \\ 4204 Golnik, Slovenia \\ ${ }^{3}$ Laboratory for Bacterial Zoonoses and Molecular Diagnostics of Bacterial Diseases, Croatian Veterinary Institute, Savska 143, \\ 10000 Zagreb, Croatia
}

Correspondence should be addressed to Mateja Pate, mateja.pate@vf.uni-lj.si

Received 14 January 2011; Revised 14 March 2011; Accepted 16 March 2011

Academic Editor: Mitchell V. Palmer

Copyright (C) 2011 Mateja Pate et al. This is an open access article distributed under the Creative Commons Attribution License, which permits unrestricted use, distribution, and reproduction in any medium, provided the original work is properly cited.

\begin{abstract}
Mycobacterium celatum, a slowly growing potentially pathogenic mycobacterium first described in humans, is regarded as an uncommon cause of human infection, though capable of inducing invasive disease in immunocompromised hosts. According to some reports, a serious disease due to M. celatum may also occur in individuals with no apparent immunodeficiency. In animals, an M. celatum-related disease has been described in three cases only: twice in a domestic ferret (Mustela putorius furo) and once in a white-tailed trogon (Trogon viridis). In this paper, we report the first detection of M. celatum in a domestic pig (Sus scrofa domestica) and roe deer (Capreolus capreolus). A nation-wide overview of human M. celatum infections recorded in Slovenia between 2000 and 2010 is also given. Pulmonary disease due to M. celatum was recognized in one patient with a history of a preexisting lung disease.
\end{abstract}

\section{Introduction}

M. celatum, a slowly growing potentially pathogenic mycobacterium, was first described in humans in 1993 [1]. Its biochemical characteristics and colony morphology are similar to those of M. avium complex, M. xenopi, $M$. malmoense and $M$. shimoidei [1]. Three types of M. celatum have been identified on the basis of $16 \mathrm{~S} r D N A$ sequence heterogeneity $[1,2]$, and the possibility of having two different $16 S r R N A$ genes within the genome has been indicated [3]. Diagnostics of $M$. celatum can be challenging as the organism can be misidentified as $M$. tuberculosis due to false positive nucleic acid probe test results [4-6]. Unambiguous identification is, therefore, provided by mycolic acid high-performance liquid chromatography or nucleic acid sequencing $[1,2]$.
M. celatum is an uncommon cause of human infection, but it may induce invasive disease in immunocompromised hosts. It has been reported to cause infection mainly in persons affected by AIDS [7-11]. However, a growing list of reports indicates that a serious disease due to M. celatum may occur also in non-HIV-infected patients $[6,12-18]$.

Up to date, only three cases of M. celatum infection have been reported in animals: two in a domestic ferret (Mustela putorius furo) $[19,20]$ and one in a white-tailed trogon (Trogon viridis) [21].

In the present work, we report the first detection of $M$. celatum in a domestic pig (Sus scrofa domestica) and roe deer (Capreolus capreolus). In addition, a nation-wide overview of human M. celatum infections recorded in Slovenia between 2000 and 2010 is given. 


\section{Materials and Methods}

2.1. Animal Isolates. The first isolate was obtained in 2000 from a pooled specimen of lymph nodes of pigs $(n=30)$. Granulomatous changes on the submandibular lymph nodes were visible during routine tuberculosis monitoring at slaughterhouse. The second isolate was obtained in 2010 from a roe deer in the scope of a study on tuberculosis in wild animals. No gross lesions characteristic of tuberculosis were observed on the specimens collected from roe deer (Table 1).

2.2. Human Isolates. A total of $21 \mathrm{M}$. celatum isolates from seven patients (two males, five females) were recorded in Slovenia in the period from 2000 to 2010. HIV status of the affected persons was unknown except for one patient who was confirmed to be $H I V$-negative (Table 2(b)). According to the low prevalence of AIDS $(<0.1 \%$ in 2009) [23] in Slovenia and the age of the patients, HIV infection was regarded as unlikely for the majority of them. All the isolates were collected and identified at the National Reference Laboratory for Mycobacteria of the University Clinic Golnik in the scope of routine diagnostics of $\mathrm{TB}$ and diseases caused by nontuberculous mycobacteria in humans. With the exception of one, all the isolates were cultured from sputum specimens. An overview of the human isolates included in this study is shown in Table 2(b).

2.3. Histopathology. Specimen of pig lymph nodes was subjected to Ziehl-Neelsen (ZN) and hematoxylin-eosin staining for the histopathological examination.

2.4. Bacteriology and Identification (Animal Isolates). Specimen smears were ZN stained and examined microscopically for the presence of acid-fast bacilli (AFB). Specimens of animal tissues were processed according to the protocol described by Kent and Kubica [24] and inoculated onto the following selective media: Löwenstein-Jensen (one slant supplemented with pyruvate and one slant supplemented with glycerin), Stonebrink, Middlebrook $7 \mathrm{H} 10$ and BBL MGIT (Mycobacteria Growth Indicator Tube, Becton Dickinson, USA). Smears of the grown bacteria were ZN stained to confirm that they comprised AFB. Identification of the isolates was based on a combination of phenotypic characteristics and molecular methods. Growth rate, growth at different temperatures, and colony morphology were recorded. According to the phenotypic features, the isolates were subjected to PCR amplification targeting the insertion sequences characteristic of $M$. avium. DNA extracted from the selected colonies was subjected to IS1245 and FR300 PCR amplification employing primer sets and protocols described previously $[25,26]$. In addition, a hybridization assay using GenoType Mycobacterium CM and AS culture identification kits (Hain Lifescience, Germany) was performed according to the manufacturer's instructions. The pig isolate was subjected to $16 S$ rDNA sequencing at the National Reference Center for Mycobacteria of the Research Center Borstel in Germany. To determine the phylogenetic affiliation of the roe deer isolate, PCR using mycobacteria-specific primer pair 285/264 targeting $16 S$ rRNA gene [27] was performed. Ribosomal PCR amplicons were purified from agarose gels after the electrophoretic separation using PureLink Quick Gel Extraction Kit (Invitrogen, USA) following the manufacturer's instructions and were sequenced in both directions (Macrogen, Korea). The retrieved sequence fragments were submitted to megablast classification accessed through NCBI website (http://www.ncbi.nlm.nih.gov/).

2.5. Bacteriology and Identification (Human Isolates). Specimens from humans were auramine stained and examined by fluorescent microscope for AFB. All specimens were processed according to the protocol described by Vestal [28] and inoculated onto two solid media (Löwenstein-Jensen and Stonebrink) and into BACTEC MGIT 960 liquid medium (Becton Dickinson, USA). The following phenotypic characteristics were investigated: growth rate, growth at different temperatures, microscopic examination of colony morphology on Middlebrook 7H10 agar plates, and biochemical activity. Biochemical tests performed are summarized in Table 3. Identification with the GenoType Mycobacterium CM and AS culture identification kits (Hain Lifescience, Germany) was also performed, following the manufacturer's instructions. Details on the identification tests used in human isolates are given in Table 2(b).

\section{Results}

3.1. Pig Isolate. Bacterial growth appeared on three of the five inoculated media (Table 1). Growth was first detected after 19 days in liquid medium; nine days later, smooth filamentous (Figure 1) yellow pigmented colonies appeared on solid medium. PCR tests for $M$. avium gave negative results. Initially, the isolate was identified as a member of the genus Mycobacterium as diagnostic tests employed in our laboratory at the time of investigation did not enable identification of various nontuberculous mycobacteria to the species level. In 2003, GenoType Mycobacterium culture identification kit was introduced into laboratory routine work as a tool for improved identification of mycobacteria. As a result of retrospective investigation, the pig isolate was identified as M. celatum and was sent to the National Reference Center for Mycobacteria of the Research Center Borstel, Germany, for DNA sequencing which confirmed the isolate's identity (data on type were not available). Histopathological examination of the pig lymph node specimen revealed $\mathrm{ZN}$ negative partially incalcinated incapsulated granulomas.

3.2. Roe Deer Isolate. Bacterial growth occurred after 34 days on three media inoculated with the lymph node material. PCR tests for $M$. avium were negative. Using GenoType Mycobacterium CM and AS culture identification kits and DNA sequencing, the isolate was identified as M. celatum. Sequencing showed a duplication of electropherogram peaks after the reported insertion of additional $\mathrm{T}$ in position 214 [3]. Clear sequencing patterns were obtained after manual correction that revealed the presence of two different 


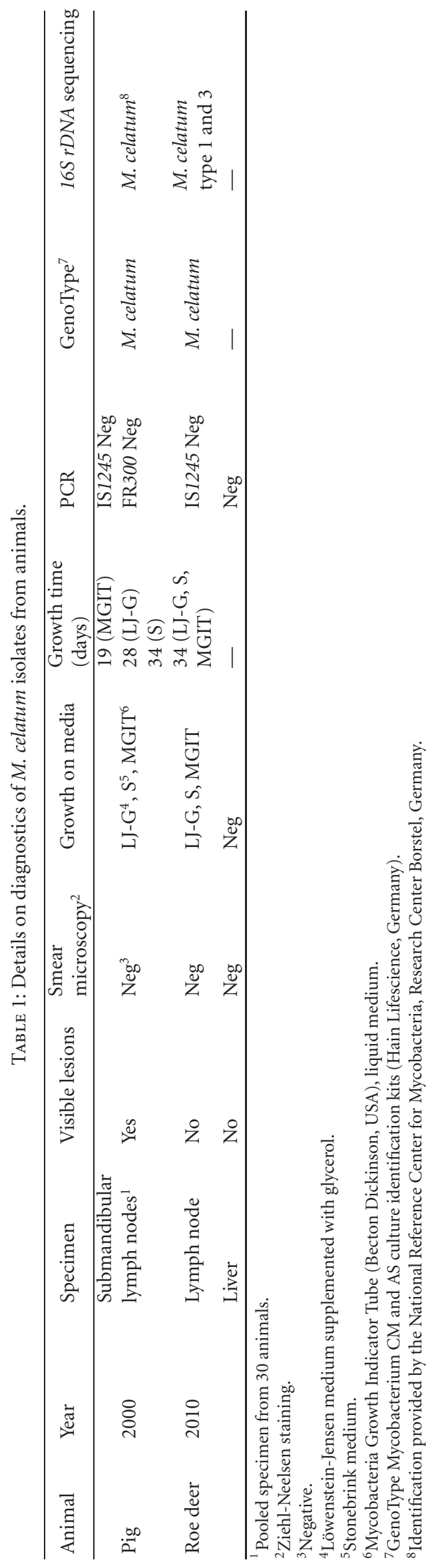


TABLE 2

(a) An overview of laboratory diagnostics of mycobacteria in humans in Slovenia between 2000 and 2010

\begin{tabular}{|c|c|c|c|c|c|c|c|}
\hline Year & No. of specimens & M. tuberculosis & M. bovis & M. caprae & M. bovis BCG & $\mathrm{NTM}^{\mathrm{a}}$ & M. celatum \\
\hline 2000 & 17016 & 1022 & 0 & 0 & 0 & 180 & 0 \\
\hline 2001 & 17576 & 1298 & 0 & 1 & 0 & 182 & 0 \\
\hline 2002 & 16062 & 1178 & 0 & 0 & 2 & 170 & 2 \\
\hline 2003 & 15236 & 1132 & 0 & 0 & 1 & 213 & 2 \\
\hline 2004 & 12268 & 972 & 0 & 0 & 3 & 136 & 0 \\
\hline 2005 & 11556 & 923 & 0 & 0 & 0 & 211 & 1 \\
\hline 2006 & 11753 & 852 & 0 & 1 & 2 & 207 & 2 \\
\hline 2007 & 11995 & 874 & 0 & 2 & 0 & 182 & 2 \\
\hline 2008 & 11566 & 889 & 0 & 0 & 0 & 178 & 5 \\
\hline 2009 & 11855 & 817 & 0 & 0 & 1 & 299 & 2 \\
\hline $2010^{\mathrm{b}}$ & 12118 & $n a^{c}$ & $\mathrm{na}^{\mathrm{c}}$ & $\mathrm{na}^{\mathrm{c}}$ & $\mathrm{na}^{\mathrm{c}}$ & $n a^{c}$ & 5 \\
\hline Total $^{\mathrm{b}}$ & 149001 & $\mathrm{na}^{\mathrm{c}}$ & $\mathrm{na}^{\mathrm{c}}$ & $\mathrm{na}^{\mathrm{c}}$ & $\mathrm{na}^{\mathrm{c}}$ & $\mathrm{na}^{\mathrm{c}}$ & 21 \\
\hline
\end{tabular}

${ }^{a}$ Nontuberculous mycobacteria.

${ }^{\mathrm{b}}$ Laboratory diagnostics of specimens received in 2010 are still ongoing; as the final reports are not yet available at the time of writing, only the number of investigated specimens is given.

${ }^{\mathrm{c}}$ Not available yet.

(b) Details on diagnostics of M. celatum from humans

\begin{tabular}{|c|c|c|c|c|c|c|c|c|c|}
\hline Patient & Gender & Year of birth & $\begin{array}{l}\text { No. of } \\
\text { isolates }\end{array}$ & $\begin{array}{c}\text { Year of } \\
\text { isolation }{ }^{1}\end{array}$ & Sample & Microscopy $^{2}$ & $\begin{array}{l}\text { Growth on } \\
\text { media }\end{array}$ & Identification & Treatment \\
\hline A & Female & 1912 & 1 & 2002 & Sputum & -3 & $\mathrm{LJ}^{6}$ & $\begin{array}{c}\mathrm{CM}^{10}, \mathrm{BC}^{11} \\
\mathrm{GT}^{12}\end{array}$ & No \\
\hline B & Female & 1947 & 1 & 2002 & Sputum & - & $\mathrm{MGIT}^{7}$ & $\mathrm{CM}, \mathrm{BC}, \mathrm{GT}$ & No \\
\hline $\mathrm{C}^{*}$ & Female & 1929 & 2 & 2003 & Sputum & - & MGIT & CM, BC, GT & No \\
\hline $\mathrm{D}$ & Male & 1959 & 1 & 2005 & Sputum & - & MGIT & $\mathrm{CM}, \mathrm{BC}, \mathrm{GT}$ & No \\
\hline $\mathrm{C}^{*}$ & Female & 1929 & 2 & 2006 & Sputum & - & MGIT & $\mathrm{CM}, \mathrm{GT}$ & No \\
\hline $\mathrm{C}^{*}$ & Female & 1929 & 2 & 2007 & Sputum & $\pm^{4}$ & $\begin{array}{l}\text { LJ, ST }^{8}, \\
\text { MGIT }\end{array}$ & $\mathrm{CM}, \mathrm{GT}$ & No \\
\hline $\mathrm{C}^{*}$ & Female & 1929 & 5 & 2008 & Sputum & $\pm^{5}$ & LJ, ST, MGIT & $\mathrm{CM}, \mathrm{GT}$ & Yes \\
\hline $\mathrm{E}$ & Female & 1932 & 1 & 2009 & $\begin{array}{l}\text { Bronchial } \\
\text { aspirate }\end{array}$ & - & $\begin{array}{l}\text { Banič liquid } \\
\text { medium }^{9}\end{array}$ & $\mathrm{CM}, \mathrm{GT}$ & No \\
\hline $\mathrm{F}$ & Male & 1971 & 1 & 2009 & Sputum & - & LJ, MGIT & $\mathrm{CM}, \mathrm{GT}$ & No \\
\hline $\mathrm{C}^{*}$ & Female & 1929 & 4 & 2010 & Sputum & - & LJ, ST, MGIT & $\mathrm{CM}, \mathrm{GT}$ & Yes \\
\hline G & Female & 1974 & 1 & 2010 & Sputum & - & MGIT & $\mathrm{CM}, \mathrm{GT}$ & No \\
\hline
\end{tabular}

${ }^{*}$ Patient with M. celatum pulmonary disease and a history of tuberculosis and M. avium and M. intracellulare-related pulmonary mycobacteriosis; confirmed as $H I V$-negative.

${ }^{1}$ No M. celatum isolates were identified in years 2000, 2001, and 2004.

${ }^{2}$ Auramin fluorescent staining.

${ }^{3}$ Negative.

${ }^{4}$ One specimen positive and one specimen negative for acid-fast bacilli.

${ }^{5}$ Four specimens positive and one specimen negative for acid-fast bacilli.

${ }^{6}$ Löwenstein-Jensen medium.

${ }^{7}$ Mycobacteria Growth Indicator Tube (Becton Dickinson, USA), liquid medium.

${ }^{8}$ Stonebrink medium.

${ }^{9}$ In-house liquid medium containing human serum (described in [22]).

${ }^{10}$ Colony morphology.

${ }^{11}$ Biochemical tests (for details, see Table 3).

${ }^{12}$ GenoType Mycobacterium CM and AS culture identification kits (Hain Lifescience, Germany).

$16 S$ rRNA gene copies (i.e., type 1 and type 3 ) in the genome of M. celatum (Table 1).

3.3. Human Isolates. Auramine staining generated positive results in five out of 21 specimens from which M. celatum was later cultivated. All smear positive specimens belonged to one patient with pulmonary disease due to M. celatum. The vast majority of isolates grew in liquid medium; in eight out of 21 cases, the liquid medium was the only medium in which bacterial growth was detected. Colonies that appeared 
TABLE 3: Results of biochemical tests performed on human $M$. celatum isolates collected between 2002 and $2005^{1}$.

\begin{tabular}{lc}
\hline Test & Result \\
\hline Semiquantitative catalase $37^{\circ} \mathrm{C}$ & $<45 \mathrm{~mm}$ of foam \\
Catalase $68^{\circ} \mathrm{C}$ & + \\
Tween hydrolysis ( 5 days $/ 10$ days $)$ & $-/-$ \\
Arylsulfatase $(3$ days $/ 14$ days) & $+/+$ \\
Tellurite reduction & + \\
Iron uptake & - \\
Tolerance to $5 \% \mathrm{NaCl}$ & - \\
Tolerance to $\mathrm{TCH}(10 \mu \mathrm{g} / \mathrm{mL})$ & + \\
Growth on $\mathrm{MacConkey} \mathrm{agar}$ & - \\
Growth at $25^{\circ} \mathrm{C}$ & + \\
Growth at $30^{\circ} \mathrm{C}$ & + \\
Growth at $37^{\circ} \mathrm{C}$ & + \\
Growth at $45^{\circ} \mathrm{C}$ & - \\
Growth rate & Slow \\
Colony morphology $($ Middlebrook $7 \mathrm{H} 10$ agar $)$ & Smooth \\
\hline
\end{tabular}

${ }^{1}$ From the beginning of 2006, identification of the isolates did not include biochemical tests any more. Five M. celatum isolates from the period 2002-2005, on which biochemical tests were performed, displayed uniform biochemical characteristics shown in this table.

${ }^{2} \mathrm{TCH}$ : thiophene 2-carboxylic hydrazide.

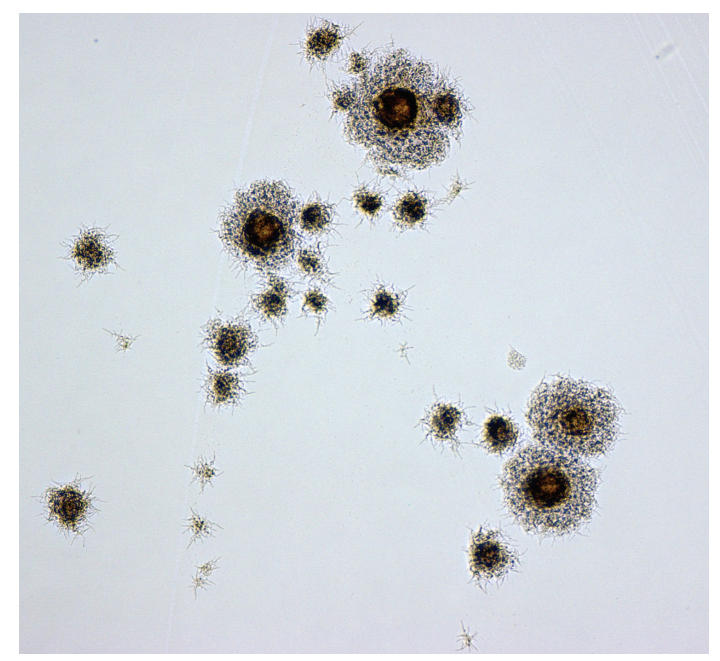

FIGURE 1: Colony morphology of pig M. celatum isolate grown on Middlebrook 7H10 agar plates and viewed under the microscope $(\times 100)$.

on solid media were small and smooth. Until the end of 2003, the identification was based on phenotypic methods (colony morphology under stereomicroscope, biochemical tests). However, they were not discriminative enough and the isolates were identified as nonchromogenic mycobacteria. Biochemical features of the investigated isolates are shown in Table 3 and are consistent with the characteristics listed in the first description of the species [1]. GenoType Mycobacterium $\mathrm{CM}$ and AS culture identification kits were introduced into routine work in 2004 and have been used since for the identification of all investigated mycobacterial isolates to the species level. The isolates of unknown mycobacterial species cultured between 2000 and 2003 were identified in retrospective. The results of bacteriological and molecular investigations of the human isolates are summarized in Table 2(b).

\section{Discussion}

In the first reports on M. celatum in animals, the organism was identified as the aetiological agent of disseminated granulomatous infections. The findings related to the infection ranged from classical tuberculous lesions with well-defined granulomas in the white-tailed trogon [21] to poorly defined granulomas described in the ferret [19]. The lesions present in the trogon were identical as those seen in classical avian mycobacteriosis caused by M. avium [21]. The route of infection was not determined although the lesions in the respiratory tract indicated transmission by inhalation [19, 21]. The source of infection was also unknown; however, as most mycobacteria are ubiquitous environmental saprophytes [29], exposure to soil or water seems to be the most probable source. The presence of M. celatum in the environment has been demonstrated by its isolation from a biofilm in an aquarium [30] and by the detection of M. celatum-like DNA sequence from the soil [31]. The last paper on M. celatum infection in animals concerns splenitis in a ferret. Common occurrence of M. celatum in ferrets could be explained by the supposition that they are more susceptible to mycobacterial infections as many mycobacteria, for example, M. bovis, M. microti, M. avium, M. triplex, $M$. fortuitum, M. florentinum, $M$. interjectum, and $M$. intracellulare, have been isolated from this animal species [32-34].

In the animal specimens investigated in this study, no AFB were detected by $\mathrm{ZN}$ staining of the tissue smears. Previous papers on M. celatum infection in animals report positive smear microscopy, but low numbers of AFB were recorded in some instances $[20,21]$. The growth times of the bacteria from pig and roe deer were in between the growth times reported previously. Bacterial growth from the pig lymph node specimen was first detected after 19 days of incubation in liquid medium. Smooth filamentous and yellow pigmented colonies appeared after 28 days on one of the solid media. Bacterial growth from the roe deer lymph node specimen was detected in liquid and on two solid media after 34 days. For comparison, in a ferret with disseminated $M$. celatum infection, yellow colonies were visible after ten days [19] while small smooth and pale colonies from the ferret with splenitis appeared after eight weeks [20]. $M$. celatum types revealed in animals comprise type 1 in the trogon [21] and type 3 in the ferret with disseminated disease [19] while the type identified in the ferret with splenitis demonstrated close relatedness to the human $M$. celatum strains types 1 and 3 [20]. Our isolate from roe deer harbored two different 16S rRNA gene copies, that is, of types 1 and 3 .

The significance of M. celatum infection in the animals described in this paper is unclear. The presence and the location of TB-like lesions indicate that $M$. celatum is a potentially pathogenic organism for pigs which might 
get infected per os. The granulomatous changes resembled those seen in pigs infected with $M$. bovis or M. avium. Therefore, $M$. celatum infection might be important from the standpoint of making correct diagnosis. In roe deer specimens, there were no gross pathological changes seen on liver and the lymph nodes; histopathological examination was not performed. The finding of $M$. celatum in the lymph node tissue could be a result of ubiquitous nature of the organism, which is likely to be widespread in the environment. In both animal species, the isolation of M. celatum was a rare event. Among 152 mycobacteria isolated from pigs between 2000 and 2010, the majority (92.1\%) belonged to $M$. avium. A few isolates of Mycobacterium sp., M. terrae and $M$. fortuitum were also detected. $M$. celatum represented $0.67 \%$ of isolates. A more detailed overview of mycobacteria in animals in Slovenia was published recently [35]. The mycobacteria detection rate in wild animals was lower. The national study on tuberculosis in wild animals started in 2010. During this year, a total of 108 animals of various species were sampled and eight isolates of nontuberculous mycobacteria were detected. Two out of 31 roe deer were positive for mycobacteria; one isolate was identified as $M$. celatum and another as Mycobacterium sp.

$M$. celatum has been mainly recognized as the causative agent of pulmonary and disseminated infections in immunocompromised humans [16]. It may affect also immunocompetent patients $[6,12,16]$; however, some authors discussed the possibility that the host's defense failure associated with M. celatum infection suggests a possible hidden immunodeficiency rather than a true immunocompetence [14]. The review of the literature concerning $M$. celatum infection in non- $H I V$-infected patients revealed eight reports from seven countries in the past 16 years [6, 12-18]. Among nine cases of human $M$. celatum infection, three patients had a history of pulmonary TB $[14,17,18]$, one of TB lymphadenitis and chronic obstructive pulmonary disease [15], one of diabetes mellitus [13], and one was a lung transplant recipient [16] while for three patients no underlying disease was recorded $[6,12,16]$. In all patients, M. celatum caused pulmonary infection with the exception of two lymphadenitis cases in children $[12,16]$. Outcome of antibiotic treatment was favorable for five out of seven adult patients while in children a surgery (in one case supported by antibiotic treatment) resulted in regression of illness [17]. Smear microscopy was mostly positive for AFB. The methods used for the identification of the isolates involved phenotypic and molecular methods; definitive diagnosis was provided by $16 \mathrm{~S} r \mathrm{DNA}$ sequencing in the majority of cases.

During an eleven-year period, a total of $21 \mathrm{M}$. celatum isolates belonging to seven patients were discovered among 149,000 specimens from humans investigated in Slovenia (Table $2(\mathrm{a})$ ). M. celatum represented about $0.82 \%$ of isolates of nontuberculous mycobacteria identified between 2000 and 2010. With the exception of one isolate from bronchial aspirate, M. celatum isolates were cultivated from sputum. Smear microscopy (fluorescent auramine staining) was positive only in five sputa from one patient with pulmonary $M$. celatum disease. The remaining specimens were negative for AFB.
The occurrence of M. celatum in five patients, in which single sputum specimens were investigated, may be explained by the presence of this organism in the environment. The finding of these isolates could be regarded as random with no particular significance. In one patient, M. celatum was isolated from bronchial aspirate. Since bronchi are considered as a usually sterile site, the isolation of M. celatum from such a site could indicate the clinical importance of the infection. However, no further specimens of this 77-year-old patient were investigated. In order to meet the criteria for diagnosis of nontuberculous mycobacteria pulmonary disease, published by American Thoracic Society [36], several specimens should be collected within one year and evaluation of clinical and radiographic findings should be performed along with the results of bacteriology.

Therefore, among M. celatum isolates recovered from humans in Slovenia up to date, M. celatum infection was of clinical significance only in one elderly patient (born in 1929) with a history of tuberculosis (1952-1954, treated by thoracoplasty). In 2002-2003 and 2005-2006, this patient was treated also for $M$. avium-related and $M$. intracellularerelated pulmonary disease, respectively. The repeated isolation of $M$. celatum from 15 sputum specimens (among them, five smear positive) in five years confirms the clinical importance of the infection in this patient. The patient was first subjected to antibiotic therapy at the end of 2008. The outcome of the therapy is unknown as no specimens were received after the beginning of treatment course. However, the four M. celatum isolates obtained in 2010 and repeated therapy suggest recurrence of pulmonary infection. This case demonstrates that a preexisting lung disease represents an important risk factor for $M$. celatum pulmonary infection. This can be concluded also from the cases reported previously as the description of underlying diseases in $M$. celatum-infected patients often involved lung diseases, most frequently TB [17].

In conclusion, the finding of $M$. celatum in animals and humans suggests its presence in the environment. Its ability to induce TB-like lesions in pig indicates that the organism is potentially pathogenic for this animal species. In addition, $M$. celatum was recognized as the etiological agent of pulmonary infection in a patient with a history of a preexisting lung disease.

\section{Acknowledgment}

Milojka Šetina is gratefully acknowledged for skillful technical support and for conscientious archiving of mycobacterial isolates.

\section{References}

[1] W. R. Butler, S. P. O’Connor, M. A. Yakrus et al., "Mycobacterium celatum sp. nov.," International Journal of Systematic Bacteriology, vol. 43, pp. 539-548, 1993, Erratum in: International Journal of Systematic Bacteriology, vol. 43, pp. 868, 1993.

[2] T. J. Bull, D. C. Shanson, L. C. Archard, M. D. Yates, M. E. Hamid, and D. E. Minnikin, "A new group (type 3) of Mycobacterium celatum isolated from AIDS patients in the 
London area," International Journal of Systematic Bacteriology, vol. 45, no. 4, pp. 861-862, 1995.

[3] U. Reischl, K. Feldmann, L. Naumann et al., "16s rRNA sequence diversity in Mycobacterium celatum strains caused by presence of two different copies of 16S rRNA gene," Journal of Clinical Microbiology, vol. 36, no. 6, pp. 1761-1764, 1998.

[4] W. R. Butler, S. P. O’Connor, M. A. Yakrus, and W. M. Gross, "Cross-reactivity of genetic probe for detection of Mycobacterium tuberculosis with newly described species Mycobacterium celatum," Journal of Clinical Microbiology, vol. 32, no. 2, pp. 536-538, 1994.

[5] Á. Somoskövi, J. E. Hotaling, M. Fitzgerald et al., "Falsepositive results for Mycobacterium celatum with the AccuProbe Mycobacterium tuberculosis complex assay," Journal of Clinical Microbiology, vol. 38, no. 7, pp. 2743-2745, 2000.

[6] J. H. T. Tjhie, A. F. Van Belle, M. Dessens-Kroon, and D. Van Soolingen, "Misidentification and diagnostic delay caused by a false-positive amplified Mycobacterium tuberculosis direct test in an immunocompetent patient with a Mycobacterium celatum infection," Journal of Clinical Microbiology, vol. 39, no. 6, pp. 2311-2312, 2001.

[7] E. Tortoli, C. Piersimoni, D. Bacosi et al., "Isolation of the newly described species Mycobacterium celatum from AIDS patients," Journal of Clinical Microbiology, vol. 33, no. 1, pp. 137-140, 1995.

[8] C. Piersimoni, E. Tortoli, F. De Lalla et al., "Isolation of Mycobacterium celatum from patients infected with human immunodeficiency virus," Clinical Infectious Diseases, vol. 24, no. 2, pp. 144-147, 1997.

[9] F. Garcia-Garrote, M. J. Ruiz-Serrano, J. Cosin, L. Alcala, A. Ortega, and E. Bouza, "Mycrobacterium celatum as a cause of disseminated infection in an AIDS patient," Clinical Microbiology and Infection, vol. 3, no. 5, pp. 582-584, 1997.

[10] R. A. Bonomo, J. M. Briggs, W. Gross et al., "Mycobacterium celatum infection in a patient with AIDS," Clinical Infectious Diseases, vol. 26, no. 1, pp. 243-245, 1998.

[11] S. Shahdad, M. Kaur, S. Singh, and S. Khattar, "A case of pulmonary Mycobacterium celatum in an Indian AIDS patient," International Journal of Infectious Diseases, vol. 9, no. 1, pp. 62-63, 2005.

[12] G. Haase, H. Skopnik, S. Batge, and E. C. Bottger, "Cervical lymphadenitis caused by Mycobacterium celatum," Lancet, vol. 344, no. 8928, pp. 1020-1021, 1994.

[13] I. Bux-Gewehr, H. P. Hagen, S. Rusch-Gerdes, and G. E. Feurle, "Fatal pulmonary infection with Mycobacterium celatum in an apparently immunocompetent patient," Journal of Clinical Microbiology, vol. 36, no. 2, pp. 587-588, 1998.

[14] C. Piersimoni, P. G. Zitti, D. Nista, and S. Bornigia, "Mycobacterium celatum pulmonary infection in the immunocompetent: case report and review," Emerging Infectious Diseases, vol. 9, no. 3, pp. 399-402, 2003.

[15] R. McMullan, J. Xu, T. Stanley et al., "Mycobacterium celatum pulmonary infection," Ulster Medical Journal, vol. 72, no. 2, pp. 114-116, 2003.

[16] D. C. Christiansen, G. D. Roberts, and R. Patel, "Mycobacterium celatum, an emerging pathogen and cause of false positive amplified Mycobacterium tuberculosis direct test," Diagnostic Microbiology and Infectious Disease, vol. 49, no. 1, pp. 19-24, 2004.

[17] C. K. Tan, C. C. Lai, C. H. Chou, and P. R. Hsueh, "Mycobacterium celatum pulmonary infection mimicking pulmonary tuberculosis in a patient with ankylosing spondylitis," International Journal of Infectious Diseases, vol. 13, no. 6, pp. e459-e462, 2009.
[18] H.-J. Jun, N. Y. Lee, J. Kim, and W.-J. Koh, "Successful treatment of Mycobacterium celatum pulmonary disease in an immunocompetent patient using antimicobacterial chemotherapy and combined pulmonary resection," Yonsei Medical Journal, vol. 51, no. 6, pp. 980-983, 2010.

[19] M. Valheim, B. Djønne, R. Heiene, and D. A. Caugant, "Disseminated Mycobacterium celatum (type 3) infection in a domestic ferret (Mustela putorius furo)," Veterinary Pathology, vol. 38, no. 4, pp. 460-463, 2001.

[20] E. Piseddu, M. Trotta, E. Tortoli, M. Avanzi, S. Tasca, and L. Solano-Gallego, "Detection and molecular characterization of Mycobacterium celatum as a cause of splenitis in a domestic ferret (Mustela putorius furo)," Journal of Comparative Pathology, vol. 144, no. 2-3, pp. 214-218, 2011.

[21] M. Bertelsen, C. Grøndahl, and S. Giese, "Disseminated Mycobacterium celatum infection in a white-tailed trogon (Trogon viridis)," Avian Pathology, vol. 35, no. 4, pp. 316-319, 2006.

[22] S. Banic, "A new liquid medium for the primary cultivation of tubercle bacilli," Pathologia et Microbiologia, vol. 29, no. 2, pp. 243-251, 1966.

[23] UNAIDS, "Global report: UNAIDS report on the global AIDS epidemic," 2010, http://www.unaids.org/documents/ 20101123_GlobalReport_em.pdf.

[24] G. P. Kent and G. P. Kubica, Public Health Mycobacteriology. A Guide for the Level III Laboratory, US Department of Health and Human Services, Public Health Service, Centers for Disease Control, Atlanta, Ga, USA, 1985.

[25] Z. M. Kunze, F. Portaels, and J. J. McFadden, "Biologically distinct subtypes of Mycobacterium avium differ in possession of insertion sequence IS901," Journal of Clinical Microbiology, vol. 30, no. 9, pp. 2366-2372, 1992.

[26] C. Guerrero, C. Bernasconi, D. Burki, T. Bodmer, and A. Telenti, "A novel insertion element from Mycobacterium avium, IS1245, is a specific target for analysis of strain relatedness," Journal of Clinical Microbiology, vol. 33, no. 2, pp. 304-307, 1995.

[27] P. Kirschner, B. Springer, U. Vogel et al., "Genotypic identification of mycobacteria by nucleic acid sequence determination: report of a 2-year experience in a clinical laboratory," Journal of Clinical Microbiology, vol. 31, no. 11, pp. 2882-2889, 1993.

[28] A. L. Vestal, Procedures for the Isolation and Identification of Mycobacteria, Centers for Disease Control, Atlanta, Ga, USA, 1977.

[29] E. Tortoli, "Impact of genotypic studies on mycobacterial taxonomy: the new mycobacteria of the 1990s," Clinical Microbiology Reviews, vol. 16, no. 2, pp. 319-354, 2003.

[30] V. Beran, L. Matlova, L. Dvorska, P. Svastova, and I. Pavlik, "Distribution of mycobacteria in clinically healthy ornamental fish and their aquarium environment," Journal of Fish Diseases, vol. 29, no. 7, pp. 383-393, 2006.

[31] B. Z. Chilima, I. M. Clark, S. Floyd, P. E. M. Fine, and P. R. Hirsch, "Distribution of environmental mycobacteria in Karonga District, Northern Malawi," Applied and Environmental Microbiology, vol. 72, no. 4, pp. 2343-2350, 2006.

[32] J. R. Ragg, H. Moller, and K. A. Waldrup, "The prevalance of bovine tuberculosis (Mycobacterium bovis) infections in feral populations of cats (Felis catus), ferrets (Mustela furo) and stoats (Mustela erminea) in Otago and Southland, New Zealand," New Zealand Veterinary Journal, vol. 43, pp. 333-337, 1995.

[33] F. X. Emmanuel, A. L. Seagar, C. Doig, A. Rayner, P. Claxton, and I. Laurenson, "Human and animal infections with Mycobacterium microti, Scotland," Emerging Infectious Diseases, vol. 13, no. 12, pp. 1924-1927, 2007. 
[34] G. W. de Lisle, R. P. Kawakami, G. F. Yates, and D. M. Collins, "Isolation of Mycobacterium bovis and other mycobacterial species from ferrets and stoats," Veterinary Microbiology, vol. 132, no. 3-4, pp. 402-407, 2008.

[35] M. Pate, D. Kusar, M. Zolnir-Dovc, and M. Ocepek, "Mycobacteria in animals in Slovenia: an overview of the last decade," in Proceedings of the 30th Annual Congress of the European Society for Mycobacteriology, p. 128, Porto, Portugal, 2009.

[36] American Thoracic Society, "Diagnosis and treatment of disease caused by nontuberculous mycobacteria," American Journal of Respiratory and Critical Care Medicine, vol. 156, pp. S1S25, 1997. 

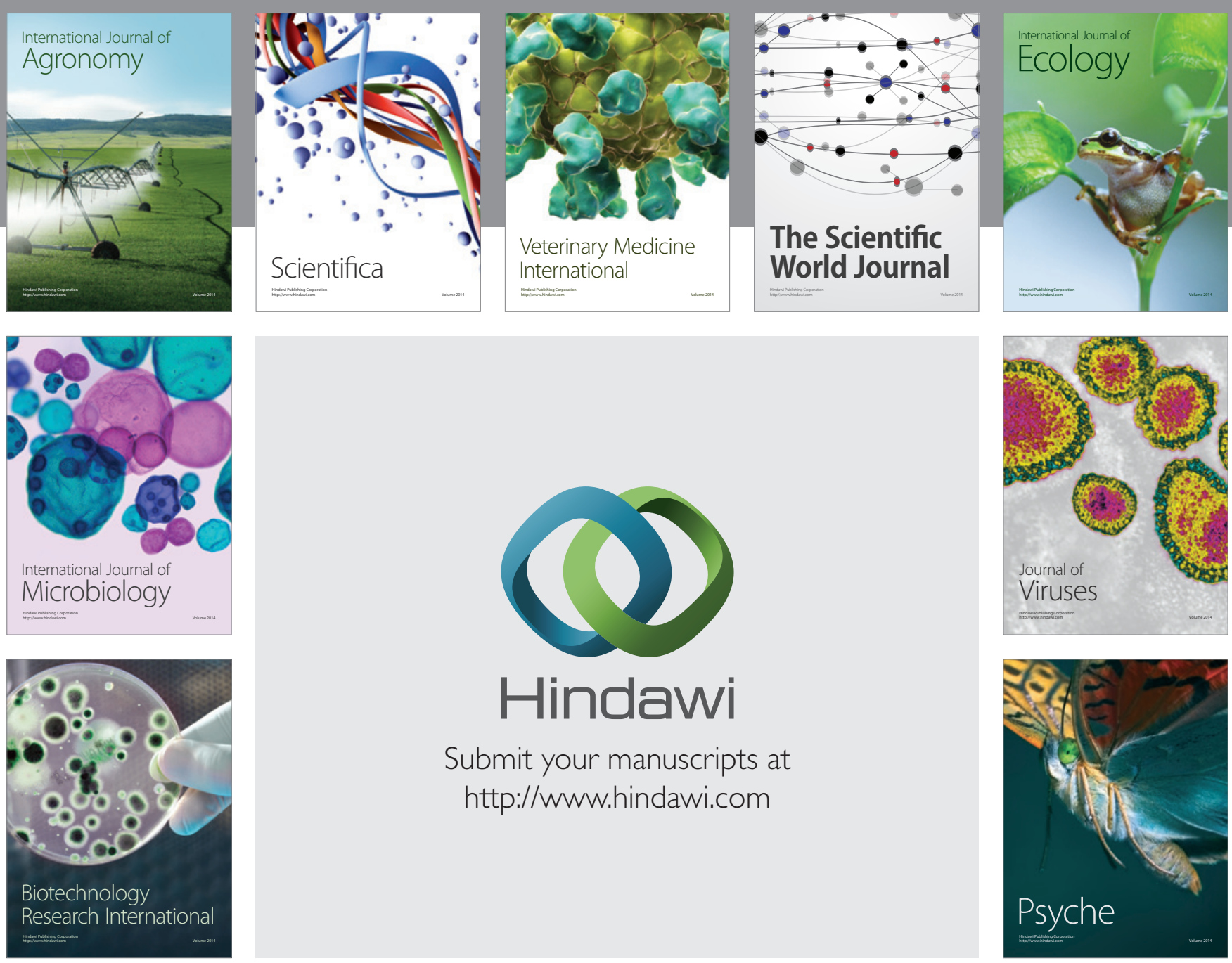

Submit your manuscripts at

http://www.hindawi.com
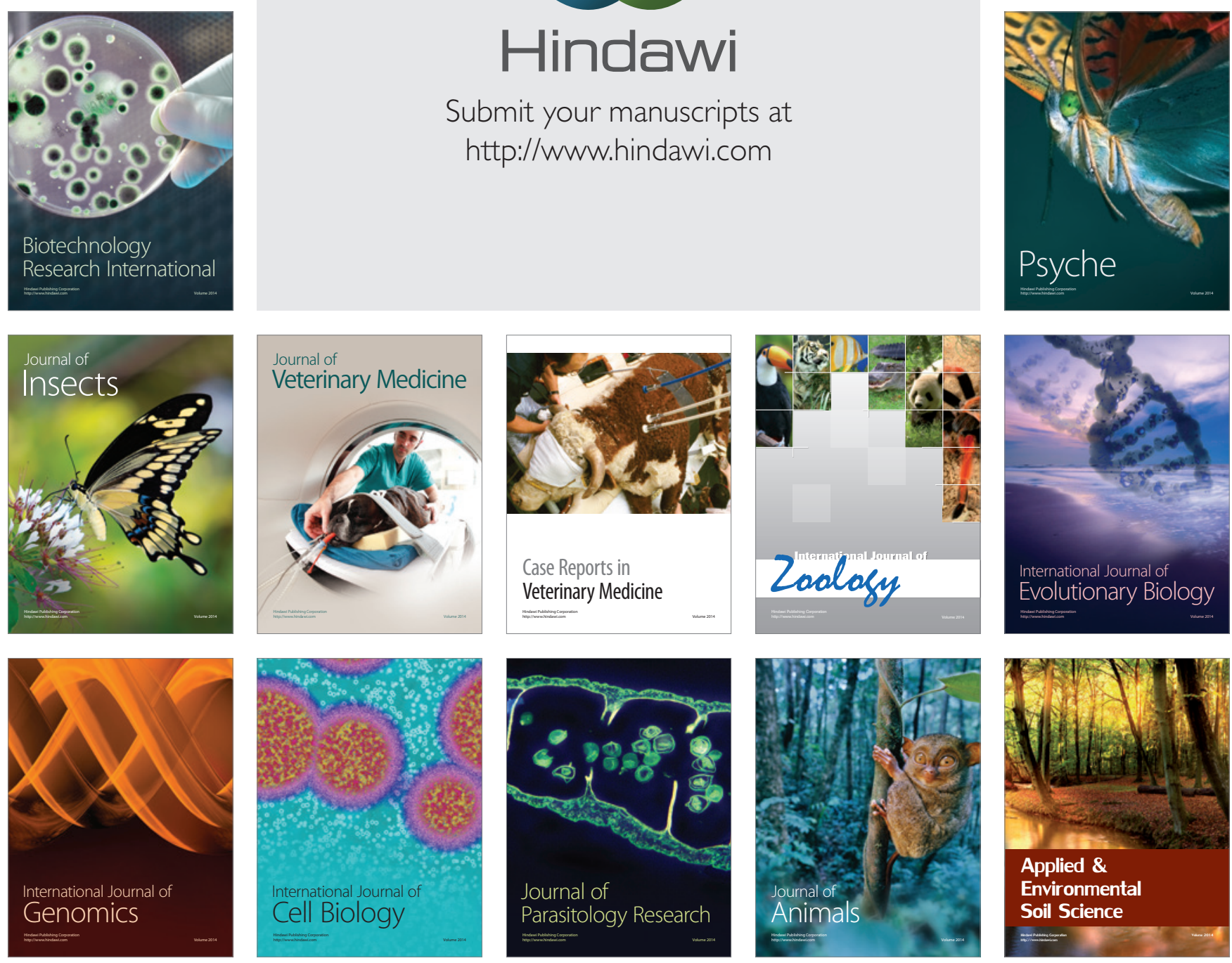